\title{
La voie vers une indemnisation ciblée du progrès médical
}

\section{Mirjam Bach}

Dr méd., experte, FMH

La médecine n'est pas une science gravée dans le marbre. Elle progresse, notamment dans la pratique clinique quotidienne. Le système de facturation doit donc pouvoir être adapté en conséquence. L'exemple de la nouvelle définition du sepsis montre comment, grâce à l'initiative de la FMH et à une coopération entre partenaires, un développement clinique peut être pris en compte de manière cohérente sur le plan tarifaire.

La définition du sepsis a déjà été adaptée à plusieurs reprises. La dernière adaptation de cette définition remonte à 2001 (sepsis 2). De plus en plus, il est apparu cependant que cette définition du sepsis en tant qu'infection avec au moins 2 des 4 critères du syndrome de réponse inflammatoire systémique (SIRS) manquait de spécificité pour poser le diagnostic à cause de la sensibilité marquée. Par ailleurs, de nouvelles découvertes scientifiques concernant le métabolisme cellulaire permettent aujourd'hui une nouvelle compréhension du sepsis. Ainsi, la réaction du corps et la dysfonction organique qui en résulte sont considérées ici comme essentielles. Un choc septique comprend des changements du système circulatoire et du métabolisme cellulaire. Ces processus sont décisifs pour une létalité plus élevée et ont des conséquences plus graves qu'une simple hypotension réfractaire au traitement. Ces nouvelles découvertes nécessitaient une nouvelle définition. La conférence de consensus a donc décrit en 2014 les critères du sepsis 3 comme suit: infection (suspectée ou avérée) accompagnée d'une dysfonction organique avec au moins deux points du score SOFA (Sequential Organ Failure Assessment). Cette nouvelle interprétation du sepsis vise à identifier plus rapidement et avec plus de précision les patients à risque et à élargir les possibilités de traitement. La composition du score SOFA est la suivante:

Sequential (Sepsis-related) Organ Failure Assessment Score ${ }^{a}$

\begin{tabular}{|c|c|c|c|c|c|}
\hline & SCORE & & & & \\
\hline SYSTEME D'ORGANES & 이 & 1) & 2 & 3 & \\
\hline \multicolumn{6}{|l|}{\begin{tabular}{|l|} 
Respiration \\
\end{tabular}} \\
\hline $\mathrm{PaO}_{2} / \mathrm{FiO}_{2}, \mathrm{mmHg}(\mathrm{kPa})$ & $\geq 400(53.3)$ & $<400(53.3)$ & $<300(40)$ & \begin{tabular}{|l|}
$<200(26.7)$ à support \\
respiratoire
\end{tabular} & $\begin{array}{l}<100(13.3) \text { à support } \\
\text { respiratoire }\end{array}$ \\
\hline \multicolumn{6}{|l|}{ Coagulation } \\
\hline Plaquettes, $\times 10^{3} / \mu \mathrm{L}$ & $\geq 150$ & $<150$ & $<100$ & $<50$ & $<20$ \\
\hline \multicolumn{6}{|l|}{ Foie } \\
\hline Bilirubine, mg/dL ( $\mu \mathrm{mol} / \mathrm{L})$ & $<1.2(20)$ & 1.2-1.9 (20-32) & $2.0-5.9(33-101)$ & $6.0-11.9(102-204)$ & $>12.0(204)$ \\
\hline \multicolumn{6}{|l|}{\begin{tabular}{|l|} 
Cardiovasculaire \\
\end{tabular}} \\
\hline Pression artérielle moyenne & MAP $\geq 70 \mathrm{mmHg}$ & MAP $<70 \mathrm{mmHg}$ & \begin{tabular}{|l|} 
dopamine $<5$ ou \\
dobutamine \\
(indépendamment de \\
la dose)
\end{tabular} & \begin{tabular}{|l|} 
dopamine $5.1-15$ ou \\
adrénaline $\leq 0.1$ ou \\
noradrénaline $\leq 0.1^{\circ}$
\end{tabular} & $\begin{array}{l}\text { dopamine }>15 \text { ou } \\
\text { adrénaline }>0.1 \text { ou } \\
\text { noradrénaline }>0.1^{\mathrm{b}}\end{array}$ \\
\hline \multicolumn{6}{|l|}{ Système nerveux central } \\
\hline Glasgow Coma Scale score ${ }^{c}$ & 15 & $13-14$ & $10-12$ & $6-9$ & $<6$ \\
\hline \multicolumn{6}{|l|}{\begin{tabular}{|l|} 
Rénal \\
\end{tabular}} \\
\hline $\begin{array}{l}\text { Créatinine, } \mathrm{mg} / \mathrm{dL}(\mu \mathrm{mol} / \mathrm{L}) \text { ou } \\
\text { Excrétion urinaire, } \mathrm{mL} / \mathrm{d}\end{array}$ & $<1.2(110)$ & $1.2-1.9(110-170)$ & $2.0-3.4(171-299)$ & $\begin{array}{l}3.5-4.9(300-440) \text { ou } \\
<500 \mathrm{ml} / \mathrm{d}\end{array}$ & $\begin{array}{l}>5.0(440) \text { ou } \\
<200 \mathrm{ml} / \mathrm{d}\end{array}$ \\
\hline
\end{tabular}

"Adapté de Vincent et al"

Dose administrée des catécholamines en $\mu \mathrm{g} / \mathrm{kg} / \mathrm{min}$ pendant au moins une heure

'Glasgow Coma Scale Score de 3 à 15

${ }^{27}$ Vincent JL, Moreno R, Takala J, et al; Working Group on Sepsis-Related Problems of the European Society of Intensive Care Medicine. The SOFA (Sepsis-related Organ Failure Assessment) score to describe organ dysfunctionffallure. Intensive Care Med. 1996:22(7):707-710. 


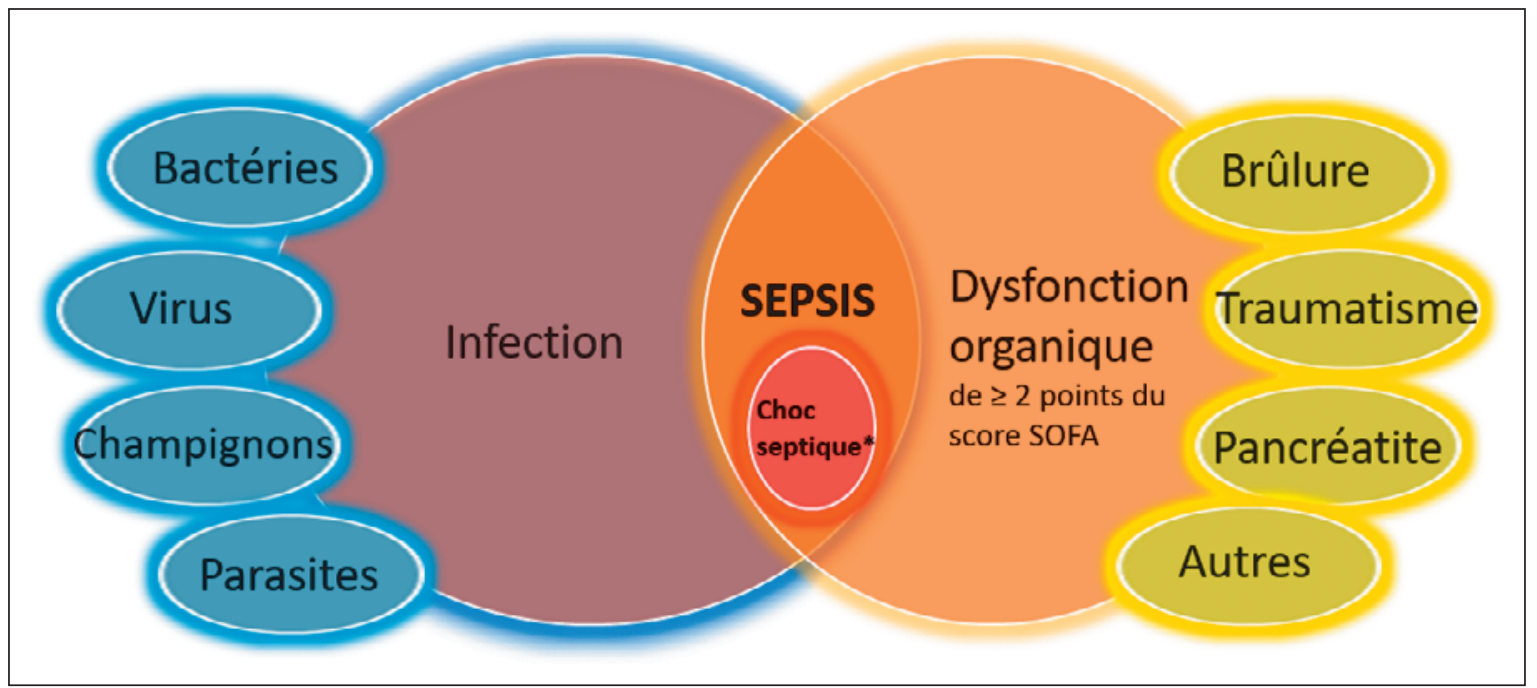

Relation entre infection, sepsis et dysfonctionnement organique (Source: Deutsches Ärzteblatt, adapté de Weis et al. [1])

* implique une hypotension réfractaire au remplissage vasculaire et une lactatémie de plus de 2 mmol/l après substitution volémique adéquate

La nouvelle définition du sepsis tend à s'imposer dans la pratique clinique. Il convient par conséquent de tenir compte de ce progrès clinique aussi dans le système tarifaire.

\section{La prise en compte du sepsis dans le codage}

La prise en compte adéquate d'un cas clinique dans le système tarifaire nécessite une interaction ciblée de tous les acteurs concernés. Dans le domaine hospitalier, SwissDRG SA adapte chaque année le système de forfaits par cas SwissDRG sur la base de données. Un

\section{Une prise en compte appropriée des cas de sepsis n'est possible qu'en cas de congruence des bases de codage.}

logiciel de groupage effectue l'attribution d'un cas clinique au DRG (Diagnosis-related group) correspondant. Pour ce faire, il procède à une analyse systématique des différents paramètres et établit la sévérité du cas sur la base des diagnostics principaux, des diagnostics secondaires et des traitements saisis. C'est là qu'intervient un autre acteur. L'Office fédéral de la statistique (OFS) publie les catalogues de classification pour les diagnostics (CIM-10-GM) et pour les traitements (CHOP) valables en Suisse. Dans le cadre de l'élaboration des directives de codage, l'OFS est tenu de réglementer l'application des systèmes de classification pour le logiciel de groupage. Une directive particulière définit dans le manuel de codage la façon de coder un sepsis. La combinaison suivante de diagnostics est nécessaire pour classer correctement le cas:
- diagnostic qui spécifie le foyer infectieux

- "sepsis dû au germe responsable»

- SIRS avec ou sans dysfonction organique

- le cas échéant, dysfonctions organiques qui en résultent et sont traitées

Le codage d'un SIRS d'origine infectieuse avec défaillance d'organe (code CIM-10: R65.1) a, compte tenu de la logique d'attribution des forfaits par cas, une pondération forte dans les versions actuelle et antérieures de SwissDRG. En général, la sévérité du cas et le revenu associé augmentent, car ce diagnostic implique une utilisation des ressources d'une manière significativement plus élevée.

Avec la procédure de propositions, les cantons, les assureurs, H+ Les hôpitaux de Suisse et la FMH, ou plus précisément les sociétés de discipline médicale, peuvent soumettre des propositions d'amélioration du système de forfaits par cas SwissDRG et d'adaptation des catalogues de classification et de leur utilisation. En 2016 déjà, avec l'appui de la FMH, les sociétés de discipline médicale concernées ont déposé, auprès de

La médecine moderne a besoin d'une indemnisation différenciée et d'une base de données solide.

l'OFS, une proposition de mise à jour de la directive de codage du sepsis selon la nouvelle définition. En mai 2017, les représentants de l'OFS, ceux de SwissDRG SA et les délégués des diverses sociétés concernées se sont accordés pour que la proposition soit mise en œuvre dès 2019 . 
Par la suite, SwissDRG SA a intégré le sepsis 3 dans la logique de groupage pour la version 8.0 de SwissDRG, valable dès le $1^{\text {er }}$ janvier 2019. En conséquence, cette version du diagnostic «R65.1 SRIS d'origine infectieuse avec défaillance d'organe» n'attribue aucune sévérité du cas. Ainsi, le facteur décisif est désormais le diagnostic "sepsis» ainsi que les dysfonctions d'organes à traiter. Par conséquent, l'adaptation corrélative de la directive de codage valable pour le sepsis est essentielle. Sans la congruence des deux éléments, il n'est pas possible d'indemniser adéquatement les cas de sepsis, en tenant compte de la sévérité de chacun d'eux. Tant que durera cette incongruité, il ne sera pas non plus possible d'évaluer les cas sur la base de données en vue d'optimiser le logiciel de groupage pour les versions futures.

\section{Manque de ressources à l'OFS}

L'OFS est responsable de la publication et de l'entretien des systèmes de classification. Il s'appuie pour cela sur le Groupe d'experts technique (TEEX), un comité consultatif. Ce groupe est composé de partenaires de SwissDRG SA: des assureurs, des représentants de la Conférence suisse des directrices et directeurs cantonaux de la santé (CDS), des délégués de $\mathrm{H}+$ et de la FMH. Cette dernière a obtenu, au sein des sociétés médicales concernées, une consolidation concernant une nouvelle directive de codage du sepsis. Vu le manque de ressources à l'OFS, la FMH a pris l'initiative de soumettre la directive en question au sein du TEEX, afin que l'adaptation de la version 8.0 de SwissDRG à la pratique clinique soit également prise en compte dans la facturation.

Malgré le large consensus atteint, l'OFS a décidé, en raison du manque de personnel, de ne pas intégrer la directive dans le manuel de codage 2019. Et ce, bien que l'incohérence avec le système de groupage était déjà connue. Une adaptation de la directive de codage «sepsis» a été envisagée pour 2021.

\section{La coordination et la coopération sont des prérequis fondamentaux}

Le Conseil fédéral constitue la dernière instance pour l'approbation des versions tarifaires et des directives de codage. La dernière possibilité pour éviter une attribution non spécifique, une indemnisation indifférenciée et une production de données inexploitables restait donc le recours au Conseil fédéral par le biais du conseil d'administration de SwissDRG SA. En effet, chaque version de facturation prévue doit être soumise avec les directives de codage de l'OFS au Conseil fédéral pour approbation. Le conseil d'administration de SwissDRG SA a décidé de recommander au Conseil fédéral l'adoption du projet de directive de codage du sepsis élaboré par la FMH et ses sociétés. Sous réserve de l'approbation par le Conseil fédéral de la version 8.0 de SwissDRG, la directive de codage publiée sous https://www.swissdrg.org $\rightarrow$ Somatique aiguë $\rightarrow$ Notices pour la saisie des prestations $\rightarrow$ PDF Règle de codage du sepsis remplacera la directive de codage figurant dans le manuel de codage. Ainsi finalement, grâce à la coopération interdisciplinaire, un progrès clinique pourrait bien être pris en compte rapidement dans le système tarifaire.

\section{Référence}

1 Weis S, Dickmann P, Pletz M W, Coldewey S M, Gerlach H, Bauer M. Sepsis 2017: Eine neue Definition führt zu neuen Konzepten; Dtsch Arztebl. 2017;114(29-30):A-1424-8.

Vous trouverez la directive de codage consolidée du sepsis sous: https://www.swissdrg.org $\rightarrow$ Somatique aiguë $\rightarrow$ Notices pour la saisie des prestations $\rightarrow$ PDF Règle de codage du sepsis 